\title{
El desarrollo rural a través de las aportaciones científicas-académicas en revistas españolas $(1990-2010)^{1}$
}

\author{
REMEDIOS LARRUBIA VARGAS \\ Departamento de Geografía, Universidad de Málaga \\ rlarrubia@uma.es \\ SUSANA R. NAVARRO RODRÍGUEZ \\ Departamento de Geografía, Universidad de Málaga \\ susanros@uma.es
}

Recibido: 30 de Junio de 2010

Enviado a evaluar: 5 de Julio de 2010

Aceptado: 24 de Febrero de 2011

\section{RESUMEN}

El objetivo de este trabajo es realizar una valoración cuantitativa y cualitativa de las aportaciones sobre el desarrollo rural que aparecen en las principales revistas de contenido geográfico en las dos últimas décadas. La selección comprende 20 revistas españolas que se inscriben en las disciplinas de geografía, economía y turismo. El vaciado bibliográfico ha derivado en la lectura de 77 artículos relacionados con la temática de estudio.

Palabras claves. Desarrollo rural, Leader, Proder, territorio, turismo rural.

\section{The rural development through the scientific-academic contributions in spanish journals (1990-2010)}

\begin{abstract}
The aim of this paper is to realize a valuation, both quantitative and qualitative, of the contributions focused on rural development that have been published during the last twenty years in the main journals with geographical contents. The selection has comprised 20 Spanish journals, under the subjects geography, economy and tourism, and 77 papers have been considered in ourstudy.
\end{abstract}

Keys words. Rural development, Leader, Proder, territory, rural tourism.

1 Este trabajo se inscribe en el proyecto Po7-Hum-03049 "Desarrollo metodológico sobre la evaluación de la Capacidad para usos recreativos de espacios naturales", financiado por la Consejería de Innovación, Ciencia y Empresa de la Junta de Andalucía. 
Le développement rural grâce aux contributions scientifiques et universitaires dans les revues espagnoles (1990-2000)

\section{RESUMÉ}

L'objectif de c'étude est faire une valorisation quantitative et qualitative des apports sur le développement rural dans le contexte académique de la Géographie. Pur faire ça, nous avons sélectionné les principaux vingt revues espagnoles rapportées avec les matières de Géographie, Economie et Tourisme pendant les derniers vingt ans. C'analyse a débouché dans la lecture de 77 articules rapportées avec le développement rural.

Mots clé: Developpement rural, Leader, Proder, territoire, tourisme rural.

\section{EL RECORRIDO DEL DESARROLLO RURAL: LUCES Y SOMBRAS}

A partir de la década de los ochenta se hizo manifiesta la profunda crisis del sistema rural europeo que obligó a modificar algunos de sus sistemas de protección tradicionales e hicieron patente la necesidad de incorporar otro tipo de estrategias destinadas a las zonas rurales europeas. Como consecuencia de esta nueva situación se iba haciendo evidente que, por un lado, había que actuar selectivamente en el medio rural según los problemas y características de ciertos territorios y, por otro lado, que en algunas regiones desfavorecidas el refuerzo de las estructuras no debía limitarse al sector agrario sino extenderse al conjunto económico regional. De esta manera se van dando una serie de pasos que permiten hablar de una emergente política rural europea. El objetivo de esta política ha sido la revitalización de los territorios rurales a través de la implicación de la población en las estrategias de desarrollo, de la diversificación económica de actividades, de la valorización de los recursos endógenos o del enfoque local en cuanto a la escala de actuación. Esta nueva orientación de la PAC alcanza su máxima expresión en los programas e iniciativas LEADER y PRODER, iniciativas de desarrollo rural que nacen del convencimiento de que éste debe ser integrado, endógeno y local.

En este punto, y tras el recorrido de la política de desarrollo rural, se puede apuntar que son muchos los elementos de coherencia que sustentan el conjunto de las medidas, pero también los interrogantes que puede plantear: interrogantes en torno al modelo de desarrollo propuesto, a la receptividad de las regiones atrasadas para adoptarlo y en relación a la pretendida plurifuncionalidad de los espacios rurales.

Con respecto al primer interrogante la idea que hay que destacar es que la UE ha hecho un cambio sustancial en la orientación política hacia el desarrollo rural; aunque la prioridad la sigue teniendo la tradicional política de precios y mercados, ya que sigue acaparando el mayor presupuesto. Pero la cuestión fundamental se refiere a la suficiencia de las dotaciones de los fondos estructurales, pese al esfuerzo en su incremento, éste no deja de ser modesto, en relación con la amplitud de los objetivos, además se han privilegiado las líneas de actuación de alcance regional sobre otros ejes (agricultura y el desarrollo rural).

El segundo interrogante a considerar es el propio modelo de desarrollo propuesto y la receptividad de las regiones más atrasadas para adoptarlo. Ante ello, cabe preguntarse 
¿hasta qué punto las políticas puestas en marcha se adaptan a la especificidades de cada espacio?. Por un lado, la dificultad reside en la diversidad de medios rurales existentes con marcadas diferencias territoriales y sociales, como ya lo indicaba el documento sobre el "Futuro del Mundo Rural"; por otro lado, hay que considerar que, en modo alguno, las ayudas llegan por igual a todas las regiones. Con los elementos hasta aquí expuestos cabría considerar la propia dificultad de generar el desarrollo de áreas deprimidas y la incidencia en el mismo del nivel de desarrollo de los ámbitos en que se insertan. Ciertamente, hay que reconocer que existe un alto grado de consenso sobre las causas, la naturaleza y la profundidad de la crisis del mundo rural, y no tanto sobre los mecanismos e instrumentos para hacer operativo un sistema de desarrollo rural. Quizás la conclusión a la que conduce es que se trata de un modelo que puede ser eficaz bajo circunstancias concretas y que no siempre será trasladable a otros contextos temporales, ni espaciales. Es decir, no existe un modelo universal aplicable a todos los lugares, porque ni las situaciones de partida, ni los problemas, ni los recursos o las formas de actuación son equiparables.

Con respecto a la tercera cuestión ¿hasta qué punto la multifuncionalidad se ha conseguido?, hay que apuntar que ha sido clara desde un principio la desviación de los fondos hacia el desarrollo de las actividades turísticas. Ante ello, cabe preguntarse si tanto turismo rural es perjudicial o beneficioso. Una de sus principales debilidades es, paradójicamente, la escasez de estudios que pongan en evidencia los aportes que el turismo rural está generando en el desarrollo rural. El turismo no debe sustituir a las actividades agropecuarias tradicionales sino que es una forma de ampliar y diversificar las opciones productivas de las zonas rurales. Habría que valorar hasta qué punto ha propiciado la diversificación productiva, la generación de recursos económicos directos, el mantenimiento de propiedades y la mejora de infraestructuras, además de mejorar las condiciones de vida de las familias directamente implicadas en la actividad, o el progreso y fortalecimiento de otras actividades productivas.

En este punto, podemos apuntar que en el mundo rural se da actualmente una gran paradoja y es que a pesar de las múltiples políticas, inversiones, iniciativas y programas, éste sigue estando en crisis. En esta línea, Rodríguez Martínez (2008) considera que a pesar de los progresivos e indudables avances de la política europea de desarrollo rural, la ausencia, hasta el momento, de una verdadera política europea de desarrollo rural es algo más que un simple postulado hipotético.

Al unísono de este interés político-administrativo por el espacio rural se generó otro en el ámbito académico-científico. Prueba de ello ha sido la profusión de publicaciones desde los primeros momentos en los que se plantea el viraje hacia este nuevo modelo de política comunitaria. Desde la década de los 90, la mayor parte de reuniones científicas de geógrafos, especialmente en los Coloquios de Geografía Rural, les han dedicado un espacio importante a cuestiones relacionadas con el desarrollo rural y local, tanto explícita como implícitamente. El interés científico por esta temática llevó a la creación el 27 de septiembre de 2003, en el seno de la AGE (Asociación de Geógrafos Españoles), del grupo de Trabajo de Desarrollo Local.

Haciendo un recorrido por estas reuniones científicas (Tabla 1), han sido en los Coloquios desarrollados por el grupo de trabajo de Geografia rural de la AGE (Asociación de Geógrafos Españoles) desde principios de la década de los 90 en los que más atención 
se ha prestado a estas cuestiones. En el VII Coloquio (Córdoba, 1994), si bien no existe ninguna ponencia que explícitamente recoja esta temática, sí se adaptan a esta línea algunas comunicaciones en relación al turismo rural a partir de la iniciativa Leader y el impulso del turismo en áreas rurales. Los dos Coloquios posteriores (Jaca, 1996 y Vitoria-Gasteiz 1998) recogen dos ponencias con títulos más orientados en esta línea. Pero fue en el $X$ Coloquio de Geografia Rural (Lleida, 2000) cuando se alcanzó la máxima especialización temática y profusión de trabajo a través de dos ponencias dirigidas claramente al desarrollo rural que incluyen dos subponencias: "El impacto del turismo, de las actividades recreativas y del fenómeno residencial en las áreas rurales" y "Las políticas de desarrollo rural. Evaluación de resultados y debate en torno a sus orientaciones futuras", donde más de 30 comunicaciones se desarrollaron en esta línea. En los dos coloquios posteriores (Santander, 2002 y León, 2004) se apreció una disminución en el interés por esta temática (10 comunicaciones), para volverse a reactivar en el XIII Coloquio (Baeza, 2006) a través de dos ponencias y 16 comunicaciones. Los dos últimos coloquios (Murcia, 2008 y Cáceres, 2010) no incluyeron ponencias específicas en esta línea, si bien indirectamente dieron cabida a comunicaciones relativas al turismo y actividades de ocio en espacios rurales.

Tabla 1. Coloquios de Geografía (1990-2010)

\begin{tabular}{|c|c|c|}
\hline CONGRESOS & PONENCIAS & COMUNICACIONES \\
\hline VII Col. Ga Rural (Córdoba, 1994) & $\begin{array}{l}\text { 1.- Medio físico, actividad agraria } \\
\text { y medio ambiente } \\
\text { 2.-Espacios naturales protegidos: } \\
\text { propiedad, actividad agraria y ocio }\end{array}$ & $\begin{array}{l}2 \\
3\end{array}$ \\
\hline $\begin{array}{l}\text { VIII Col. Ga Rural (Jaca, Zaragoza, } \\
\text { 1996) }\end{array}$ & $\begin{array}{l}\text { 1.-Desarrollo Rural en Zona de } \\
\text { Montaña }\end{array}$ & 11 \\
\hline IX Col. Ga Rural (Vitoria-Gasteiz, 1998) & $\begin{array}{l}\text { 1.- El postproductivismo en los espa- } \\
\text { cios rurales }\end{array}$ & 8 \\
\hline X Col. Ga Rural (Lleida, 2000) & $\begin{array}{l}\text { 1.- Las nuevas funciones socioeco- } \\
\text { nómicas de los espacios rurales } \\
\text { 2.-Políticas de planificación y de } \\
\text { desarrollo de los espacios rurales }\end{array}$ & $\begin{array}{l}13 \\
20\end{array}$ \\
\hline XI Col. Ga Rural (Santander, 2002) & $\begin{array}{l}\text { 1.- Los procesos de urbanización } \\
\text { en el medio rural } \\
\text { 2.- Tradición, renovación e innova- } \\
\text { ción en los usos y aprovechamien- } \\
\text { tos en las áreas rurales de montaña }\end{array}$ & $\begin{array}{l}2 \\
4\end{array}$ \\
\hline XII Col. Ga Rural (León, 2004) & $\begin{array}{l}\text { 1.- Mujer y movimientos migrato- } \\
\text { rios en espacios rurales } \\
\text { 2.- Los espacios rurales inventados } \\
\text { ¿conservación o canalización? }\end{array}$ & $\begin{array}{l}1 \\
3\end{array}$ \\
\hline XIII Col. Ga Rural (Baeza, 2006) & $\begin{array}{l}\text { 1.- El sector agrario español y su } \\
\text { adaptación a la política agraria } \\
\text { comunitaria en los últimos } 20 \text { años. } \\
\text { 2.- Las políticas comunitarias de } \\
\text { D.R. y su incidencia sobre las regio- } \\
\text { nes españolas } \\
\text { 3.- El futuro de los espacios rurales } \\
\text { europeos ante las recientes reformas } \\
\text { de la política agraria comunitaria }\end{array}$ & $\begin{array}{l}2 \\
10 \\
4\end{array}$ \\
\hline XIV Col. Ga Rural (Murcia, 2008) & 1.- Urbanización en el medio rural & 2 \\
\hline XV Col. Ga Rural (Cáceres, 2010) & $\begin{array}{l}\text { 1.- Paisajes y economías agrarias } \\
\text { 2.- Patrimonio rural }\end{array}$ & $\begin{array}{l}1 \\
3\end{array}$ \\
\hline
\end{tabular}

Fuente: Actas de Congresos Nacionales de Geografía y Coloquios de Geografía rural 


\section{OBJETIVOS Y METODOLOGÍA}

El repaso de los trabajos recogidos en los distintos Coloquios geográficos sobre la nueva orientación de la política de la Unión Europea nos llevó a plantearnos la necesidad y la oportunidad de realizar un análisis de la misma en las principales revistas de contenido geográfico, una vez que el camino andado sobre estas cuestiones podemos considerarlo avanzado. Se pretende realizar una reflexión de conjunto, una puesta a punto de carácter teórico, sobre las aportaciones científicas-académicas al desarrollo rural, en definitiva vislumbrar qué tipo de balance se hace sobre el conjunto de estas medidas, con relación al objetivo global de desarrollo rural.

Dada la magnitud de la tarea que debíamos abordar, y en aras a racionalizar esfuerzos y ser operativos, hemos realizado una doble selección, por un lado de carácter temporal, optando por los trabajos en torno al desarrollo rural y al papel que juega en él el turismo rural a partir de 1990, momento crucial en el cambio de concepción política de la UE y, por otro lado, atendiendo al lugar de publicación; de esta forma se ha realizado el vaciado de revistas nacionales de reputado prestigio y nivel científico en las disciplinas que se han ocupado tradicionalmente de las materias que estábamos considerando, fundamentalmente Geografia, Turismo y Economía. El resultado ha sido el vaciado de 20 revistas, donde se han contabilizado 77 artículos relativos al desarrollo y al turismo rural (Tabla 2 y Anexo bibliográfico).

Tabla 2. Procedencia de los artículos analizados

\begin{tabular}{|l|l|}
\hline TÍTULO DE LAS REVISTAS & NÚMERO DE ARTÍCULOS \\
\hline Actualidad Leader & 12 \\
\hline Agricultura y Sociedad & 2 \\
\hline Anales de Geografía & 1 \\
\hline Baetica & 2 \\
\hline Boletín de la A.G.E. & 5 \\
\hline Cuadernos de Geografía & 5 \\
\hline Cuadernos de Turismo & 9 \\
\hline Cuadernos Geográficos & 1 \\
\hline Documents d'anàlisi geogràfica & 2 \\
\hline Ería & 2 \\
\hline Estudios Geográficos & 1 \\
\hline Geographicalia & 3 \\
\hline Investigaciones Geográficas & 5 \\
\hline Norba & 6 \\
\hline Papeles de Economía Española & 3 \\
\hline Papeles de Geografía & 1 \\
\hline Revista Española de Estudios Agrosociales y Pesqueros & 1 \\
\hline Revista de Estudios Agrosociales & 5 \\
\hline Revista de Estudios Regionales & 5 \\
\hline Revista de Estudios Turísticos & 6 \\
\hline Total Revistas y artículos & 77 \\
\hline
\end{tabular}

El mayor número de trabajos aparece en la Revista de divulgación del Ministerio de Agricultura y Pesca denominada Actualidad Leader (12 publicaciones). Le siguen Cuadernos de Turismo (9 artículos), Revista de Estudios Regionales y Norba, ambas con 
6 publicaciones. Estas cuatro revistas reúnen el $42,85 \%$ de todos los trabajos consultados, mientras que en las 16 revistas restantes aparecen el 57,16\% de los trabajos. Las revistas que menos espacio han dedicado a las temáticas que estamos estudiando, con sólo una aportación, son Anales de Geografia, Cuadernos Geográficos, Estudios Geográficos, Papeles de Economía y Revista Española de Estudios Agrosociales y Pesquero.

En cada una de las mencionadas aportaciones hemos centrado el análisis en el conocimiento de las fuentes, de las temáticas generales abordadas, de los aspectos concretos tratados, de los objetivos declarados si los hubiere de dichos estudios, y de la metodología utilizada. Hemos querido comprobar cuáles son las reflexiones expresadas sobre el desarrollo y el turismo rural, los enfoques y estrategias utilizadas, la oportunidad de dichos trabajos y los impactos de los mismos, pero indudablemente también estábamos interesados en detectar, si existieran, elementos recurrentes, carencias y limitaciones.

Para organizar el análisis de los trabajos, en una primera aproximación, hemos identificado cinco líneas temáticas, las cuales se subdividirán en otros tantos subapartados en función de su contenido. Tres de las cinco líneas temáticas concentran el $88,29 \%$ de los trabajos: "Los programas Leader, tanto a nivel teórico como aplicado", al incluir el 46,75\% de los artículos, se convierte en la temática más ampliamente tratada; "Aspectos conceptuales sobre desarrollo y turismo rural" y "El turismo rural a nivel territorial", reúnen cada una de ellas un 22,09\% de las publicaciones. Los trabajos relacionados con "El desarrollo rural a nivel territorial" (7,79\%) y "Turismo rural y género" (3,89\%) constituyen las dos líneas temáticas menos recurrentes.

Respecto a los 10 subapartados reconocidos, los que engloban un mayor interés, traducido en una mayor cantidad de publicaciones, son "Planteamientos teóricos-conceptuales sobre el desarrollo rural" y el "Turismo rural en el contexto español", con 13 artículos cada una, seguidas de los "Aspectos teóricos y descriptivos de las Iniciativas de Desarrollo, Leader y Proder" (10 artículos) y el estudio de la "Iniciativa Leader en España" (9 trabajos). El análisis territorial reúne a un 50\% de los artículos y los estudios de carácter teórico son un $29,87 \%$ del conjunto analizado (Tabla 3 ).

Tabla 3. Líneas y sublíneas temáticas.

\begin{tabular}{|c|c|c|c|c|c|}
\hline LINEAS TEMÁTICAS & $\mathrm{N}^{\mathrm{o}}$ & $\%$ & SUBLİNEAS TEMÁTICAS & $\mathrm{N}^{\mathrm{o}}$ & $\%$ \\
\hline \multirow{4}{*}{$\begin{array}{l}\text { 1.- Iniciativas de desarrollo } \\
\text { y Proder): Teoría y Práctica }\end{array}$} & \multirow{4}{*}{36} & \multirow{4}{*}{46.75} & $\begin{array}{l}\text { a.- Aspectos teóricos y descriptivos de las } \\
\text { Iniciativas de desarrollo }\end{array}$ & 10 & 12.98 \\
\hline & & & b.- Iniciativa Leader en España & 9 & 11.68 \\
\hline & & & c.- Leader y Proder a nivel subestatal & 11 & 14.28 \\
\hline & & & d.- Turismo rural y programas Leader & 6 & 7.79 \\
\hline \multirow{2}{*}{$\begin{array}{l}\text { 2.- Aspectos conceptuales s } \\
\text { desarrollo y el turismo rura: }\end{array}$} & \multirow{2}{*}{16} & \multirow{2}{*}{20.77} & $\begin{array}{l}\text { a.- Planteamientos teóricos-conceptuales } \\
\text { sobre el desarrollo rural }\end{array}$ & 13 & 16.88 \\
\hline & & & $\begin{array}{l}\text { b.- Planteamientos teóricos-conceptuales } \\
\text { sobre el turismo rural }\end{array}$ & 3 & 3.89 \\
\hline \multirow[t]{2}{*}{ 3.- Turismo rural a nivel terı } & \multirow[t]{2}{*}{16} & \multirow[t]{2}{*}{20.77} & $\begin{array}{l}\text { a.- Nuevas modalidades de turismo en } \\
\text { espacios rurales }\end{array}$ & 3 & 3.89 \\
\hline & & & b.- Turismo rural en el contexto español & 13 & 16.88 \\
\hline 4.- Turismo rural y género & 3 & 3.89 & & 3 & 3.89 \\
\hline \multirow[b]{2}{*}{ 5.- Desarrollo rural a nivel $t \epsilon$} & \multirow[b]{2}{*}{6} & \multirow[b]{2}{*}{7.79} & a.- Desarrollo rural a nivel regional & 3 & 3.89 \\
\hline & & & $\begin{array}{l}\text { b.- Desarrollo rural en espacios naturales } \\
\text { protegidos }\end{array}$ & 3 & 3.89 \\
\hline TOTAL & \multicolumn{2}{|l|}{77} & 77 & & \\
\hline
\end{tabular}


Los trabajos han ido surgiendo de manera creciente y a medida que se ha ido cerrando cada una de las etapas de las iniciativas de desarrollo rural puestas en marcha. Hay constancia del interés por esta temática desde 1991, siendo a partir del año 1995 cuando se asiste a una mayor profusión de resultados, para luego decrecer a finales de la primera década del 2000. Los trabajos han sido realizados mayoritariamente por geógrafos, amén de alguna participación de investigadores procedentes de las áreas de economía, sociología agraria y algunos realizados por parte de los técnicos de la administración encargados de la materialización de las políticas de desarrollo rural.

Las fuentes más utilizadas se pueden dividir en tres grandes categorías. En primer lugar, fuentes documentales y bibliográficas (Diario Oficial de las Comunidades Europeas, Planes Estratégicos, Documentos comunitarios, legislaciones vigentes, etc); en segundo lugar, fuentes estadísticas (Instituto Nacional de Estadística, Instituto de Estudios Turísticos, Unidad Española del Observatorio Europeo Leader, Guías oficiales de hoteles y camping, Anuarios Estadísticos, MAPA, Consejerías de Agricultura y Diputaciones provinciales, OMT, Guías de Turismo rural, etc); y, por último, trabajo de campo (encuestas y entrevistas a Grupos de Acción Local, CEDER, empresas, usuarios, etc).

\section{PLANTEAMIENTOS ACADÉMICOS-CIENTÍFICOS DEL DESARRO- LLO RURAL}

Las investigaciones sobre el desarrollo rural llevadas a cabo, especialmente por geógrafos en las dos últimas décadas, incluyen un amplio espectro de trabajos de temáticas múltiples que intentaremos delimitar. Aspectos históricos, teóricos, coyunturales, prácticos han dado lugar a investigaciones individuales o colectivas dentro del interés sobre el espacio rural multifuncional donde ya no siempre la agricultura es la actividad principal. El contenido científico de los artículos lo hemos agrupado en las cinco líneas temáticas antes señaladas, así como en los subapartados diferenciados (Tabla 3 y Anexo Bibliográfico).

\section{LÍNEA 1. INICIATIVAS DE DESARROLLO (LEADER Y PRODER): TEORÍA Y PRÁCTICA}

El análisis de los programas de diversificación y dinamización de actividades en el medio rural (Leader y Proder) constituye una temática muy recurrente desde que se operó el cambio de política comunitaria. Son trabajos en los que se analiza, de forma más cuantitativa que cualitativa, la iniciativa Leader en primer lugar, sobretodo en su versión I y II, y en menor medida el programa Proder, especialmente en la primera fase.

La idea que se asume como punto de partida en todos estos trabajos es la profunda transformación vivida por el mundo rural, en este caso el español, y que la emergente política rural europea está trazando nuevas vías de desarrollo que buscan dar respuestas a las exigencias de revitalización y expansión de esas zonas. Igualmente es generalizada la concienciación de que las actuales estrategias de desarrollo rural deben ser integradas, endógenas y locales, haciendo hincapié en la importancia de los procesos de identidad territorial como catalizadores de las iniciativas de desarro- 
1lo. Por último, se enfatiza la importancia de las iniciativas Leader y Proder y el papel revitalizador económico que posee el turismo rural.

Los 36 artículos que se incluyen en esta línea de trabajo, cuyo contenido en general es relativamente semejante, se pueden agrupar en varias áreas algo diferenciadas.

a.- Aspectos teóricos y descriptivos de las iniciativas de desarrollo. Los 10 trabajos que corresponden a esta línea de análisis se centran exclusivamente en el análisis de las iniciativas Leader, con especial incidencia en sus dos primeras versiones. En líneas generales, los esquemas de trabajo suelen ser muy parecidos. Se trata de análisis meramente descriptivos de los programas sin realizar ningún planteamiento prospectivo de lo que pueden aportar en la dinamización de las áreas rurales. Los aspectos más recurrentes en los que se detienen la inmensa mayoría de ellos son:

- Evolución de las teorías sobre el desarrollo en general y el desarrollo rural en particular, resaltando la génesis y el método del desarrollo rural con enfoque local (Sancho, 1999; Cebrián, 2003).

- La construcción social en el desarrollo local, y la importancia del capital social en los procesos de desarrollo y cómo, en este sentido, el programa Leader es un referente (Giménez y Pérez, 1994), así como un tema central para el estudio de carácter sociológico; en algunos casos se han determinado sucintamente los tipos y características de las redes creadas en algunos grupos Leader (Buciéga, 2005)

- El carácter participativo, endógeno e integrado del nuevo desarrollo rural. En este caso se analizan las causas que determinan la existencia o no de participación, se plantea cómo el punto de partida en la mayoría de las ocasiones ha sido negativo, y que la necesaria difusión para fomentarla ha sido poco eficiente (Prats y Blanco, 1998).

- La justificación de la necesidad de la continuidad de las acciones emprendidas tras la culminación de las distintas fases previas también ha sido objeto de atención de algunos investigadores (Martínez de Bujanda, 1998; Sancho, 1999).

- Ha sido muy habitual resaltar las conexiones de los Leader con los logros del turismo rural (Velasco, 2002).

- Por último, cabe destacar, dentro de este subapartado temático, un conjunto de trabajos que, por sus planteamientos diferentes al grueso de los incluidos en este grupo, presentan un carácter novedoso. Se trata de trabajos en los que teniendo en cuenta el momento de madurez en los que se encuentran las iniciativas de desarrollo se aboga por la necesidad de evaluar los resultados (Viñas, 2004; Esparcia y Noguera, 2000) y las dificultades que ello conlleva. En ellos se exponen el proceso metodológico de la evaluación (definición de objetivos, criterios e indicadores) y el glosario de métodos existentes, especialmente cualitativos, para evaluar proyectos de desarrollo rural y su necesidad de aplicarlos a los programas Leader. La conclusión suele poner de manifiesto la falta de cultura evaluativa existente y la poca utilidad que se desprende de las evaluaciones reglamentarias llevadas a cabo. 
b.- Iniciativa Leader en España. Los 9 artículos que se incluyen en esta línea de trabajo parten de un análisis descriptivo, bastante completo, del contexto donde se enmarcan los programas de desarrollo rural, de sus aspectos metodológicos y propositivos, para continuar con el análisis territorial de las iniciativas, desde distintas perspectivas: por un lado, a través del balance global de cada una de las iniciativas Leader objeto de análisis para todo el territorio español; por otro lado, presentando al unísono los resultados de las tres iniciativas (Febles et al 2005); y, por último, se abordan cada una o el conjunto de las iniciativas para todo el territorio español a nivel de Comunidades Autónomas (Beltrán, 1991). A partir de datos muy generales se señala la distribución de los grupos de desarrollo local, el coste de las inversiones realizadas, el origen de la financiación (comunitaria, nacional, regional o privado), los programas desarrollados en cada medida, etc. El análisis descriptivo y cuantitativo es lo habitual, careciendo de balance de las iniciativas durante el período de aplicación o sus perspectivas de futuro.

c.- Leader y Proder a nivel subestatal. Son 11 los artículos que abordan la temática Leader y Proder en ámbitos territoriales muy específicos del territorio español, que van desde la comarca o provincia (zonas de montaña en general, montaña andaluza y alicantina, provincias de Granada, Málaga y Jaén) hasta la Comunidad Autónoma (Comunidades aragonesa, gallega, castellano-leonesa y andaluza). Dentro de la línea habitual en la que se estructuran los trabajos que versan sobre las iniciativas de desarrollo, éstos también incorporan los antecedentes de las políticas de desarrollo rural en la UE, para ello se retrotraen hasta la política de socioestructura, la indemnización compensatoria y la Ley de Agricultura de Montaña (Esparcia, 1995, Rodríguez, 2000), e incluso analizan la aplicación de estas políticas en sus propios territorios (Comunidades andaluza y valenciana). A continuación, presentan una breve semblanza de la concepción, origen y características de los programas de desarrollo rural, complementándose con lo que ha sido la aplicación de las iniciativas de desarrollo en todo el territorio español y en el marco europeo (Ruiz, 1995). El siguiente paso es el análisis socio-económico y demográfico de las áreas donde se han aplicado las iniciativas, señalando las carencias y necesidades de las comunidades rurales, aunque sin olvidar los factores de oportunidad que ofrecen el territorio (Ricardo, 2003). El núcleo de los trabajos lo constituye la evaluación de los programas de desarrollo rural: distribución de proyectos e inversiones por ejes y promotor, movilización de capital por medidas, proyectos por sectores, etc (Frutos et al., 2000; Márquez et al., 2006; Larrubia y Navarro, 2003; Hidalgo, et al., 1995).

Las reflexiones finales van en la línea de lo oportuno de dar continuidad a las acciones Leader y Proder en España. Los más críticos se cuestionan el proceso de selección de las zonas, el extraordinario desvío del presupuesto hacia el turismo rural y su relación con el objetivo de la pluriactividad, la escasa evaluación de los grupos habidas hasta el momento en el que se centra el análisis, etc (Barke, 1995).

Por último, dentro de esta línea de análisis hay que destacar algunos trabajos porque presentan estructuración, objetivos y metodologías que se apartan de la generalidad. Se trata de los trabajos de Esparcia (2000), Noguera (2002), Pérez y López (2005), Márquez et al. $(2005,2006)$. En unos casos hacen un balance reflexivo sobre 
los logros y las deficiencias de los Leader a nivel español, al contrario de lo habitual, pensando más en los procesos que en las cifras. En otros casos, reflexionan en torno al grado de supervisión de la iniciativa, el impacto de ésta sobre el empleo y lo que hubiera ocurrido en las zonas rurales si no hubiera aparecido Leader. Por último, otros intentan establecer categorías de comarcas o zonas rurales acogidas a las iniciativas de desarrollo en base a dos parámetros, el grado de eficacia (proyectos respecto a la población y superficie) y el de eficiencia (inversión respecto a la población y la superficie) en materia de turismo.

d.- Turismo rural y programas Leader. El punto de partida de los seis trabajos que se incluyen en este subapartado considera que el turismo rural tiene un notable protagonismo en las acciones emprendidas por las administraciones de distintos ámbitos territoriales, prestándose especial atención el programa Leader (Sotelo, 2002, Aparicio, 2004, Yagüe, 1998, etc). La mayoría de estos trabajos "bajo el título de turismo rural" no hacen más que un análisis cuantitativo de los programas Leader. $\mathrm{Su}$ estructura habitual se mueve dentro de la línea de análisis de estos programas, con la única diferencia que reserva un apartado a examinar y valorar el efecto del programa Leader en el turismo rural, el cual "por las características socioeconómicas de las áreas rurales" se presupone que puede convertirse en un medio para sustentar su desarrollo. Los estudios que se apartan de esta línea presentan un análisis DAFO o de costes-beneficios del turismo rural, o se centran en el análisis tradicional de la oferta y demanda de los servicios turísticos (Sotelo, 2002; Blanco, 1994).

\section{LÍNEA 2. ASPECTOS CONCEPTUALES SOBRE EL DESARROLLO Y EL TURISMO RURAL}

La idea general que trasmite este conjunto de trabajos es la mutación producida en el mundo rural, la diversidad de áreas existentes en la UE planteando la necesidad de una definición común de dichas áreas para toda Europa y la evolución de las políticas de desarrollo rural aplicadas. Las 16 publicaciones incluidas en este apartado se pueden desagregar en dos subapartados: planteamientos teóricos, por un lado, del desarrollo rural (13) y, por otro lado, del turismo rural (3).

a.- Planteamientos teóricos-conceptuales sobre el desarrollo rural. Los aspectos más relevantes tratados en esta primera sublínea han sido:

- El papel de las redes en el desarrollo rural (Ward et al., 1997). Por un lado, se diferencian los dos modelos de desarrollo (exógenos y endógenos) que se han puesto en práctica en las áreas rurales europeas, subrayando que ambos tipos de procesos van a estar determinados por factores locales. Consideran que el desarrollo rural debe ser una compleja combinación de redes en las que se movilicen recursos, se determinen identidades y se consoliden redes de poder. De hecho plantean que los análisis de redes ofrecen herramientas metodológicas sencillas para investigar la constitución de asociaciones económicas, aunque no se indican los procedimientos a seguir para alcanzar este objetivo. Por otro lado, se presentan la creación, evolución, objetivos, fines, prioridades y áreas de actuación de la Red Española de Desarrollo Rural (García, 2006). 
- Las fases de constitución de las políticas de desarrollo rural en la UE (Calatrava, 1999; López, 2006; Martínez, 2006; Amarillo, 2006; etc) a partir de 1988, cuando la entonces CEE planteó su estrategia rural desde una perspectiva más global, no exclusivamente ligada a la PAC, pasando repaso a la primera reforma de la PAC de 1992, la Conferencia de Cork (1996), la segunda reforma (2003), etc.

- Reflexiones sobre el desarrollo sostenible (Barbero, 2004). Se plantean las dificultades y el desafío que supone conseguir un desarrollo sostenible a nivel económico, social y medioambiental. El medioambiente es un 'leitmotiv' de casi todos los programas de desarrollo local. Tanto en los diagnósticos como en los programas operativos siempre está presente, ya sea como misión y objetivo o como instrumento para el desarrollo. Dentro de la Iniciativa Leader, ciertos grupos de acción local le conceden siempre un lugar, explícita o implícitamente, en sus acciones para el desarrollo, ya que sitúan estas últimas en una perspectiva de desarrollo sostenible. Además, muchos territorios Leader abarcan una parte, cuando no la totalidad, de un parque nacional, regional o una zona protegida. Pero el interrogante que subyace es: ¿cómo se puede evaluar la competitividad medioambiental de un territorio?

- Dimensiones y factores del desarrollo rural a través de casos concretos en Andalucía, pero sin aportar información demasiado concreta. (Moscoso, 2005, Grande, 2007). Es interesante el planteamiento de que en la aplicación de los programas de desarrollo influyen factores no económicos que pueden explicar las desigualdades de los resultados de la iniciativa.

- Reflexiones sobre el papel clave de la participación que efectúa la Red Estatal de Desarrollo rural. Se resalta la importancia de la cooperación en el desarrollo rural, en concreto entre los Grupos de Desarrollo, que además es uno de los principales objetivos de la UE 2007-2013.

b.- Planteamientos teóricos-conceptuales sobre el turismo rural. En esta línea los aspectos abordados con más frecuencia han sido, siempre desde una perspectiva teórica, los siguientes: la imagen turística de los espacios rurales que se fundamenta en la pervivencia de rasgos tradicionales asociados con el mundo rural, la ambigüedad conceptual que planea sobre el turismo rural, sus aspectos negativos, los desajustes que puedan generar (volumen excesivo de oferta no reglada, concentración espacio-temporal) (Grande, 2007) y, la capacidad que posee para la diversificación de la economía rural, siendo algunos bastante cautos al señalar que, no obstante, no se le puede considerar la "panacea" a los problemas de los espacios rurales. (Ivars, 2000). También suele ser habitual un planteamiento evolutivo de la política estatal en turismo rural, señalándose las mejoras que esta actividad ha aportado al mundo rural (Alvarado, 2006). Como conclusión podemos señalar la idea de que si bien el turismo rural tiene entidad suficiente para figurar entre las actividades económicas que pueden conseguir una mayor prosperidad de las regiones desfavorecidas, sin embargo las políticas desarrolladas acuden a acciones que son recurrentes en todos los documentos analizados, ello cuestiona las respuestas adecuadas a cada ámbito local 
y si han logrado las acciones de turismo rural sus objetivos en las regiones desfavorecidas (Sarasa, 2000).

\section{LÍNEA 3. TURISMO RURAL A NIVEL TERRITORIAL}

Entre las nuevas funciones asumidas por los espacios rurales en aras al carácter postproductivista que van adquiriendo, es el turismo rural una de las actividades que más expansión está alcanzando, siendo paralelamente una de las temáticas que más interés ha suscitado entre los investigadores y que se refleja en la profusión de publicaciones.

En estos trabajos la idea que se asume como punto de partida es el papel del turismo como elemento clave en la diversificación y dinamismo económico del medio rural, siendo una de las alternativas que mejor está complementando las rentas agrarias y contribuyendo al desarrollo local, sin embargo en las investigaciones más recientes, es frecuente encontrar la idea en torno a la prudencia en el desarrollo turístico-rural y las consecuencias que puede implicar un desarrollo excesivo y sin planificación.

Esta línea temática agrupa un nutrido número de trabajos (16 en total) que analizan el desarrollo del turismo rural independientemente de las iniciativas de desarrollo rural. En ellos podemos diferenciar dos subgrupos.

a.- Nuevas modalidades de turismo en espacios rurales. Los tres artículos que reúne este subgrupo abordan tres nuevas modalidades de turismo en espacio rural que está teniendo gran aceptación: agroturismo, turismo deportivo y ambiental.

El ámbito territorial de estos trabajos va desde lo general, afectando a todo el espacio español (Martín, 1994), a lo local, situándose específicamente en el sudeste español (Alpujarras granadinas y comarca noroeste de la región de Murcia) (Ponce, 2009, Sayadi y Calatrava, 2001). Ambos, tras resaltar la importancia del turismo en el marco de la UE para alcanzar el desarrollo rural integrado, el papel e importancia de estas modalidades turísticas frente a otras en los ámbitos rurales o hacer un recorrido por la legislación regional, o por los problemas que afectan al sector, centran su atención en el análisis concreto de cada una de estas nuevas modalidades turísticas. La conclusión va en la línea de que aún son actividades muy marginales en las zonas rurales; si bien las modalidades turísticas deportiva y ambiental se pueden convertir en actividad principal de los empresarios, en cambio el agroturismo sólo es un medio de conseguir la complementariedad de las rentas. También se señala la problemática que suelen presentar en relación a la heterogeneidad de la oferta, la necesidad de la difusión de información y comercialización del producto y la necesaria regulación de las actividades deportivas-recreativas para limitar los impactos ambientales y generar modelos de desarrollo sostenibles.

b.-El turismo rural en el contexto español. La mayor parte de los 13 artículos seleccionados, previamente a centrar su atención en la actividad turística, se detienen en la línea reiterativa que hemos señalado para el estudio de las iniciativas de desarrollo rural (plurifuncionalidad, opción turística, recorrido de la política de la UE, etc). Desde el punto de vista espacial, los trabajos se centran bien en presentar la evolución y caracterización del turismo rural en todo el territorio español (Bardón, 1990, 
Galiano, 1991, etc.), bien a nivel regional (Galicia, Cataluña, Aragón, Murcia) o provincial (Soria, Alicante, Murcia).

El núcleo de los trabajos es el análisis de la actividad turística desde distintas perspectivas: en unos casos, se atiende al análisis de la demanda y la oferta de turismo rural incidiendo en su distribución provincial y las expectativas tanto de usuarios como de oferentes (Cánoves et al., 2005); en otros casos, a través del análisis comparativo de la oferta de turismo rural en España y en los principales países europeos, diferencian modelos de turismo rural (Herrera et al., 2005) y, por último, hay que señalar aquél que centra su interés en comparar la legislación española de ámbito nacional, con la de la región objeto de análisis (Región de Murcia) y éstas a su vez con la comunitaria (Millán, 1999).

En este grupo hay que destacar dos trabajos que analizan el turismo en espacios naturales, pertenecientes a las Comunidades Catalana y Valenciana (López y Antón, 1996; Montiel, 2003). En ellos se pone de manifiesto que los parques naturales se convierten en espacios idóneos para promover procesos ordenados y sostenibles de desarrollo turístico rural. Los usos turísticos ofrecen una gran oportunidad para redescubrir y reapropiarse por parte de la sociedad de los valores naturales y culturales del monte mediterráneo tras décadas de abandono, desuso y desconocimiento. Terminan con una propuesta de ordenación de los espacios forestales para su utilización con fines recreativos-turísticos, todo ello integrándolo en las políticas de desarrollo rural. Por último, señalan la resistencia existente por parte de la población local debido a la falta de tradición de la misma para llevar con éxito la autogestión.

\section{LÍNEA TEMÁTICA: TURISMO RURAL Y GÉNERO}

Esta línea aborda una temática conectada con la Geografía de Género. Como es sabido, el tema de la mujer en el medio rural ha acrecentado el interés en los últimos tiempos, interés que trasciende el análisis habitual del trabajo de la mujer en el medio rural (explotaciones agrarias, industria rural), debido a que la terciarización de la economía en este espacio le está dado nuevas oportunidades a través de la ampliación y diversificación de las ofertas turísticas. Los tres estudios se centran en dos áreas geográficas, la Región de Murcia y A Coruña.

Como punto de partida, todos las investigaciones resaltan la importancia que tiene el turismo como "yacimiento de empleo" en el ámbito de la UE y en el español con mayor especificidad, y el papel que desempeña en la incorporación de la mujer al mundo laboral desde el momento en que las tres cuartas partes de la mano de obra empleada en turismo rural en la UE es femenina (Sarasa, 1998). Si bien, también se trata de desmitificar el hecho de que el turismo rural sirva para incorporar a la mujer al trabajo remunerado, de forma que el papel que desempeña, en la mayoría de los casos, se puede asimilar al tradicional rol de ama de casa, manteniéndola invisible (Sparrer, 2003; Cánoves y Villarico, 2000).

Se analiza el papel de la mujer en relación al turismo rural desde dos perspectiva, por un lado, establecen las diferencias entre el trabajo de la mujer en el turismo rural y en el resto de las actividades turísticas (Sarasa, 1998), concluyéndose que a pesar 
que el turismo rural tiene una gran importancia en la creación de empleo éste es menor que el generado en otras modalidades turísticas (turismo litoral), debido a una oferta más restringida, a lo que hay que añadir que la demanda parece que todavía no ha creado los resortes claros para ejercer como actividad verdaderamente alternativa y no sólo como actividad complementaria. La segunda línea de análisis se centra en la tradicional línea de la geografía de género (jornada laboral, formación, profesión, tareas laborales y ámbitos productivos) exponiendo los aspectos positivos y negativos del turismo rural en las relaciones de género (Sparrier, 2003; Cánoves y Villarino 2000).

\section{LÍNEA 5. DESARROLLO RURAL A NIVEL TERRITORIAL}

Este grupo de trabajos analiza el desarrollo rural independientemente de los programas Leader. Suman un total de seis trabajos, que territorialmente se sitúan tres en comunidades autónomas (Murcia, Extremadura, País Vasco) y otros tres en espacios naturales, de estos últimos uno afecta a todo el territorio español y dos al andaluz (Sierras Subbéticas cordobesas y Parque de Doñana). Las ideas que subyacen en todos ellos son nuevamente las de la necesidad de lograr la diversificación económica de los espacios rurales como condición imprescindible para lograr la fijación de la población rural, el papel que están desempeñando las políticas desarrollas por distintas administraciones para lo que han implementado legislaciones específicas (Leyes de Desarrollo Rural, PORN, Programas Operativos, etc). Dos son las parcelas temáticas que podemos diferenciar:

a.- Desarrollo rural a nivel regional. Son trabajos donde se analizan bien los Programas Operativos que han incidido en el desarrollo rural de algunos territorios (región de Murcia), destacando la incidencia del Eje 7 (Agricultura y Desarrollo Rural) (Millán, 2007), o las Leyes de Desarrollo Rural (Alberdi, 2004). Tras plantear el marco donde se pone en marcha la política rural se termina con una breve valoración de los resultados, poniéndose de manifiesto que éstos han sido, en líneas generales, insuficientes. En esta línea de parcos resultados hay que mencionar un estudio centrado en los pequeños municipios rurales extremeños (Pérez, 2006) en el que se valora las posibilidades que tiene el desarrollo rural en los pequeños municipios aunque queda todavía un largo camino por recorrer a estos pequeños espacios, pues la política de desarrollo rural desarrollada hasta el momento ha dado resultados muy parciales.

$b$ - Desarrollo rural en espacios naturales protegidos. En todos estos trabajos se plantean como punto de partida los cambios que se han producido en la actividad agraria, pero también entre la sociedad rural y el resto de la sociedad; en lógica relación con ello se mencionan las transformaciones que se han ido produciendo en la UE y en España respecto al espacio rural y protegido en aras a lograr el desarrollo rural. Sus planteamientos permiten diferenciar dos grupos de trabajos: por un lado, el de Troitiño (1995), en el que llama la atención sobre la proliferación de estos espacios y de sus normativas correspondientes, para cuestionarse sobre cuál debe ser el sentido actual de los espacios protegidos, qué debe entenderse por conservación y desarrollo, cómo debe concebirse la planificación de estos espacios protegidos. Por otro lado, aquéllos que resaltan la necesidad de que en los espacios naturales protegidos sean compatibles la preservación de los valores ambientales con la implicación de la población rural para 
contribuir al desarrollo rural, sin embargo son conscientes de que dicho planteamiento cuenta con claras dificultades (envejecimiento, escasa cualificación y dinamismo emprendedor) (Pérez y Guzmán, 1994), por lo que la conclusión es la necesidad de un mayor conocimiento de las características de los espacios protegidos para ayudar o implicar a la población rural en su conservación (Prados y Elbersen, 1999).

\section{REFLEXIONES FINALES}

En este punto final se pretende hacer un balance sobre los logros alcanzados por el desarrollo rural a través del análisis de las investigaciones publicadas en revistas científicas, así como las lagunas de análisis apreciadas, lo que quizás pueda ser revelador del grado de madurez alcanzado por esta línea política implementada desde la UE a partir de la década de los 90 .

Como punto de partida hay que señalar, y así se pone en evidencia en todos los trabajos, los efectos positivos del cambio de orientación de la política comunitaria, desde el agrarismo al desarrollo rural, con ella se ha conseguido que haya una percepción diferente de una parte significativa de la sociedad rural con respecto a las zonas en las que viven, con respecto a los recursos existentes en las mismas y con respecto a su futuro, no obstante, y también es el "leit motiv" en muchos artículos, aún queda un largo camino por recorrer, en la línea sobre todo de la consolidación de los cambios iniciados.

En las investigaciones sobre el desarrollo rural hemos podido comprobar que existe un gran conocimiento a nivel teórico y global sobre la necesidad de acometer en las áreas rurales procesos de desarrollo rural, del camino seguido para alcanzarlo, así como de las características y objetivos del método adoptado, pero falta un análisis de las carencias y limitaciones de éste o la posibilidad de que existan modelos alternativos.

Un aspecto bastante reiterativo ha sido el análisis de la larga y profunda problemática que envuelve al mundo rural (despoblación, envejecimiento, falta de infraestructuras, de servicios, etc) y la necesidad de intentar llevar soluciones. No obstante se echa en falta el análisis de los logros reales conseguidos tras el período de bagaje de las iniciativas desarrolladas.

También ha sido habitual la idea en torno a las profundas transformaciones operadas sobre el mundo rural español gracias a estas iniciativas En el momento actual cabría pensar hasta qué punto estos cambios han sido inducidos más por la dinámica económica y agraria en general que como consecuencia de una estrategia rural, la cual se ha quedado más en los modelos teóricos y los grandes esquemas conceptuales que en la realidad y eficiencia de los múltiples programas puestos en prácticas. Se es consciente que el desarrollo rural sigue siendo una cuestión relevante cara al futuro de los espacios rurales, porque, a pesar de los esfuerzos realizados hasta el momento, no ha conseguido erradicar el conjunto de causas que orientaron esta nueva línea de actuación política, no debemos olvidar que en materia de financiación el desarrollo rural ha sido el "pariente pobre" frente a la potencia presupuestaria del "primer pilar de la PAC". 
La necesidad de seguir avanzando, mejorando y cubriendo las carencias que se han evidenciado, es un hecho común. En este sentido es un gran acierto que en aplicación de los principios aprobados en el Tratado de Ámsterdam, de la actual orientación de la política europea ha surgido un nuevo paradigma del carácter multifuncional de la actividad agraria, es decir, se establece un modelo agrario/rural. Se trata de promover una agricultura suficiente y compatible con un desarrollo rural sostenible, presentando una atención preferente a los profesionales de la agricultura y prioritaria a los titulares de explotaciones agrarias, que fueron olvidadas en los primeros virajes hacia el desarrollo rural. Sin embargo, según los expertos, este nuevo modelo agrario/rural está mostrando su fragilidad antes de terminar de ser implantado, ya que se enfrenta a una dinámica de los mercados y una realidad económica que tienen planteamientos casi antagónicos.

También han sido frecuentes los planteamientos muy teóricos dentro de las concepciones sociológicas sobre la cooperación y la creación de redes, aunque todo se ha quedado en el plano de lo abstracto, siendo poco usual la presentación de situaciones concretas. Hubiera sido interesante un análisis de las redes que se han generado, del papel que han tenido las comunidades rurales en relación a su capacidad de autogobernación, del grado en que los GDR (Grupo de Desarrollo Rural) han sido capaces de transmitir a la sociedad el método LEADER, el grado de participación real y efectiva de la población, etc. La conclusión que hemos podido extraer es que existe una deficiente inserción a nivel local de las medidas de desarrollo, agravada por la falta de interlocutores, por el desconocimiento de las medidas, el insuficiente flujo de información, la descoordinación administrativa, además por los recursos financieros insuficientes.

A lo largo de la lectura de estos trabajos ha sido difícil apreciar el pretendido enfoque territorial de las políticas de desarrollo. En ello ha podido incidir, por un lado, el que las aportaciones científicas se hayan movido más en el terreno de las cifras, de lo cuantitativo, del análisis de los documentos presentados por los diferentes GDR para conseguir las iniciativas, que en los aspectos cualitativos a través del contacto directo con los actores; por otro lado, el enfoque territorial ha sido realmente limitado, debido en parte a que sobre unas líneas estratégicas semejantes se desarrollan los programas en todo el territorio español, de modo que cuanto más generalizadas sean las medidas menor profundidad y capacidad de transformación tendrán. En este sentido, en los últimos análisis de las iniciativas de desarrollo se alude a la importancia del capital social como factor fundamental de desarrollo en las zonas rurales, señalándose la necesidad en las iniciativas venideras, de otorgarles mayor atención a lo social desde el momento en que no pueden formularse planteamientos globales válidos, sino que para cada caso habrán de analizarse las diferencias entre comunidades o poblaciones, considerando la historia previa y la idiosincrasia de cada una de ellas. Además, este aspecto es fundamental dado que la dotación financiera de estos programas es limitada y reducida si se compara con otros tipos de actuaciones y como es muy difícil de aumentar en la cuantía que se requiere habrá que poner más el acento en lo social que en las inversiones y cuadros financieros. En este sen- 
tido el desarrollo rural es aún más un proyecto que una realidad al no existir una política consolidada de desarrollo rural con enfoque territorial (Rodríguez, 2008).

A través de los trabajos el objetivo de dinamización económica de las iniciativas de desarrollo tampoco es clara. La mayoría de los análisis presuponen que las ideas y métodos Leader han tenido efectos directos sobre el empleo, si bien la constatación real de lo presupuestado es discutible. Faltaría un seguimiento de los efectos de las iniciativas, en cuanto al grado de consolidación de la inversión en cada espacio, los empleos y empresas creados y su continuidad, así como las incidencias sobre la población local (si ha propiciado su mantenimiento y crecimiento o por el contrario sigue estacionaria o decreciendo).

Aún más difícil es poder evaluar en qué grado los empleos han favorecido la diversificación de las actividades económicas. Lo que está claro ha sido la generalizada apuesta por el turismo rural, como forma de alcanzar la plurifuncionalidad de los espacios rurales. Pero la pretendida diversificación sin agricultura es algo cuestionable, hay que encontrar soluciones alternativas productivas para solucionar los conflictos entre la sociedad agraria y la necesaria diversificación, pero no sólo concentrada en algunos subsectores de servicios, haría falta generar empleo y renta contemplando muy de cerca el sector agroalimentario. La competitividad del sector agrario, allí donde es posible, debe ser fundamental. No hay que perder de vista varios hechos: en primer lugar que la agricultura es la pieza básica, a veces exclusiva de las zonas rurales; en segundo lugar, que en actividades externas a las del sector agrario las posibilidades de empleo no son ni tantas, ni tan sencillas, ni tan baratas como con frecuencia parecen suponerse; $y$, por último, no hay que olvidar el peligro que supone dejar de lado la agricultura cuando el mercado de trabajo entra en crisis, se convierte en un sector refugio. Al hilo de estas últimas reflexiones, los trabajos más cercanos a los momentos actuales no son ajenos a estas problemáticas, al igual que los últimos planteamientos de la UE. En estos trabajos se pone de manifiesto el riesgo que supone caer en la monofuncionalidad con un fuerte carácter estacional, con los riesgos que ello comporta de cara a la diversificación de actividades.

Asimismo también es difícil evaluar en qué grado estos empleos han supuesto la creación de nuevas empresas o hasta qué punto han sido autoempleo. En relación a este último aspecto cabe preguntarse ¿hasta qué punto el autoempleo es aconsejable? Uno de los peligros es que como en muchas ocasiones, suponen la vuelta a prácticas como las de trabajo a domicilio y derive en el incremento del trabajo sumergido; además. la idea del autoempleo como forma de resolver el paro es trasladar a las propias familias rurales la carga de resolver el problema del paro por sí mismas.

Por último, también ha sido escaso o nulo el planteamiento sobre " el día después” ¿qué ocurrirá cuando las subvenciones o la gestión desaparezcan?. La cuestión creemos que es la siguiente: si se mantienen las subvenciones se corre el riesgo de crear una población dependiente de las ayudas, como en otros muchos sectores de actividad, y si se retiran las subvenciones, cabe preguntarse, ¿está preparada la población para dar continuidad por sí misma a las acciones emprendidas y sobre todo poner en marcha otras nuevas?. ¿Han tenido verdaderamente un efecto demostrativo las iniciativas de desarrollo rural?. 
Desde nuestro punto de vista, creemos que aunque los trabajos son muy valiosos, sin embargo, la relevancia de los datos medios planteados como medidores de la eficacia o rentabilidad de las medidas es pequeña, no se consigue medir el impacto real de las iniciativas de desarrollo. Se tiene la impresión de que el desarrollo rural, por lo presupuestado y realizado, sigue siendo una política alternativa que necesita un mayor impulso económico y logre mayor consolidación lo generado.

El conjunto de estas advertencias o peligros no invalida experiencias de alto éxito con la aplicación de estos instrumentos. Quizás la conclusión a la que conduce es que se trata de un modelo que puede ser eficaz bajo circunstancias concretas y que no siempre será trasladable a otros contextos temporales ni espaciales. 
R. Larrubia Vargas y S. R. Navarro Rodríguez $\quad$ El desarrollo rural a través de las aportaciones ...

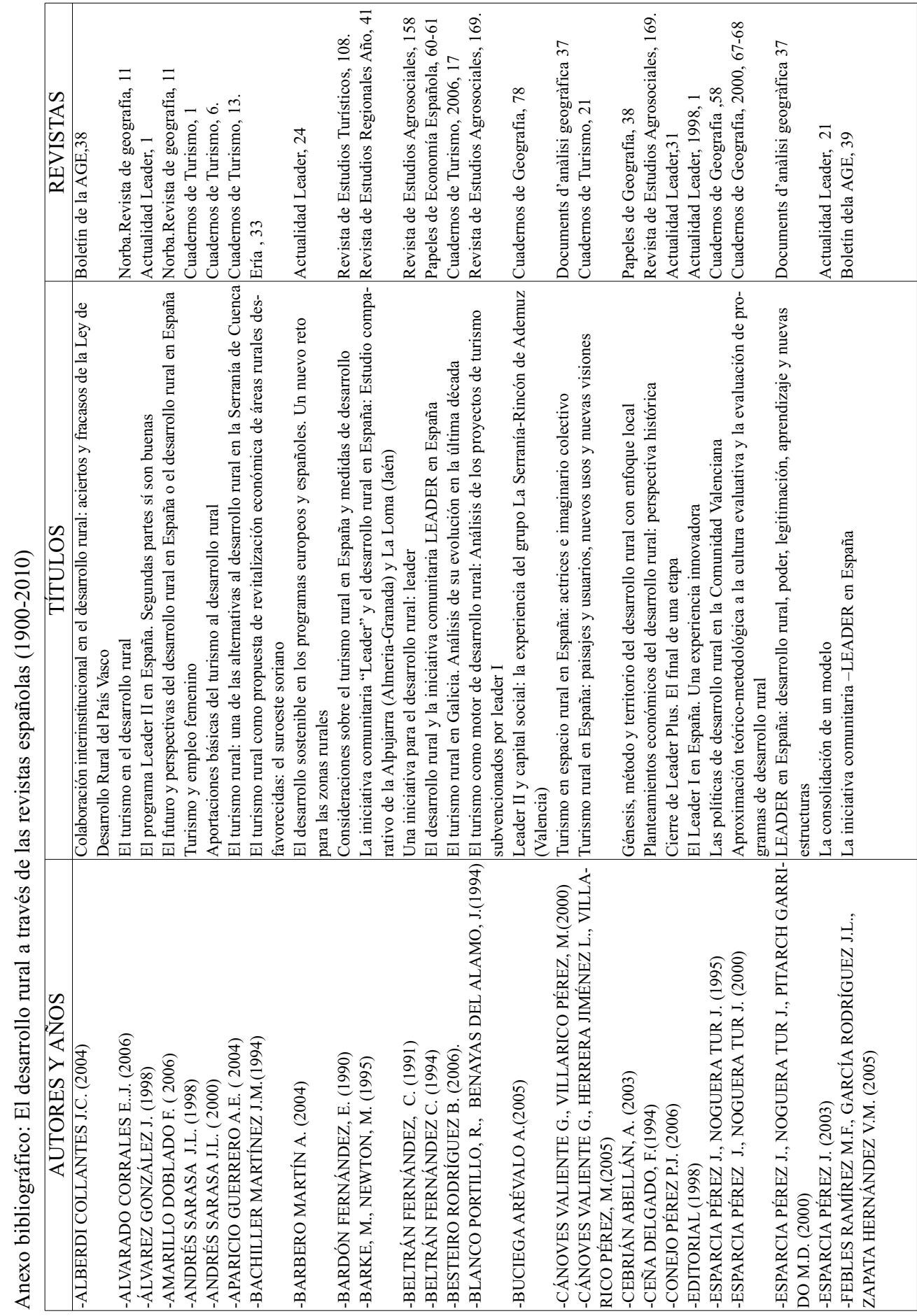

2011, vol. 31, núm. 1 61-81 


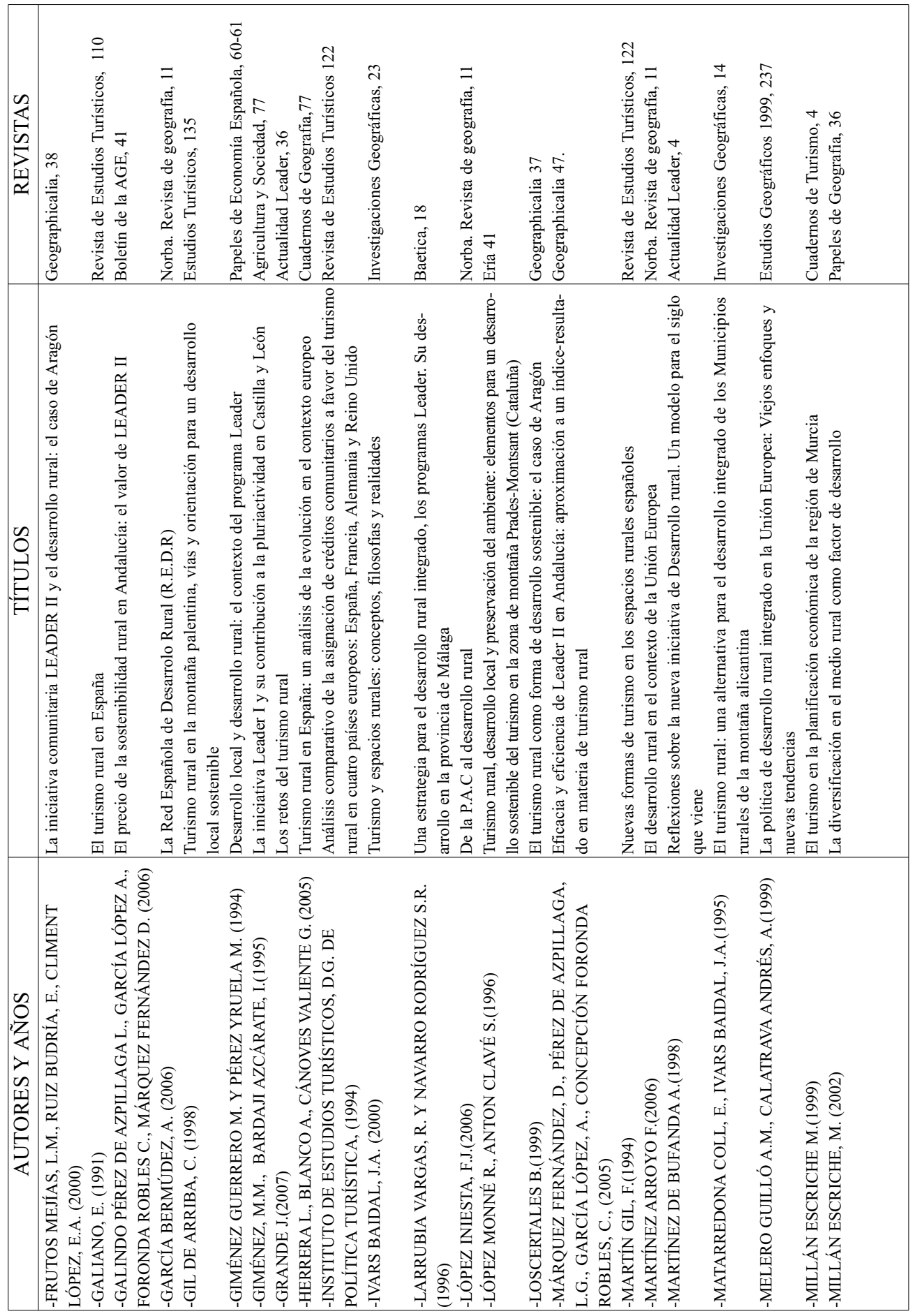


R. Larrubia Vargas y S. R. Navarro Rodríguez $\quad$ El desarrollo rural a través de las aportaciones ...

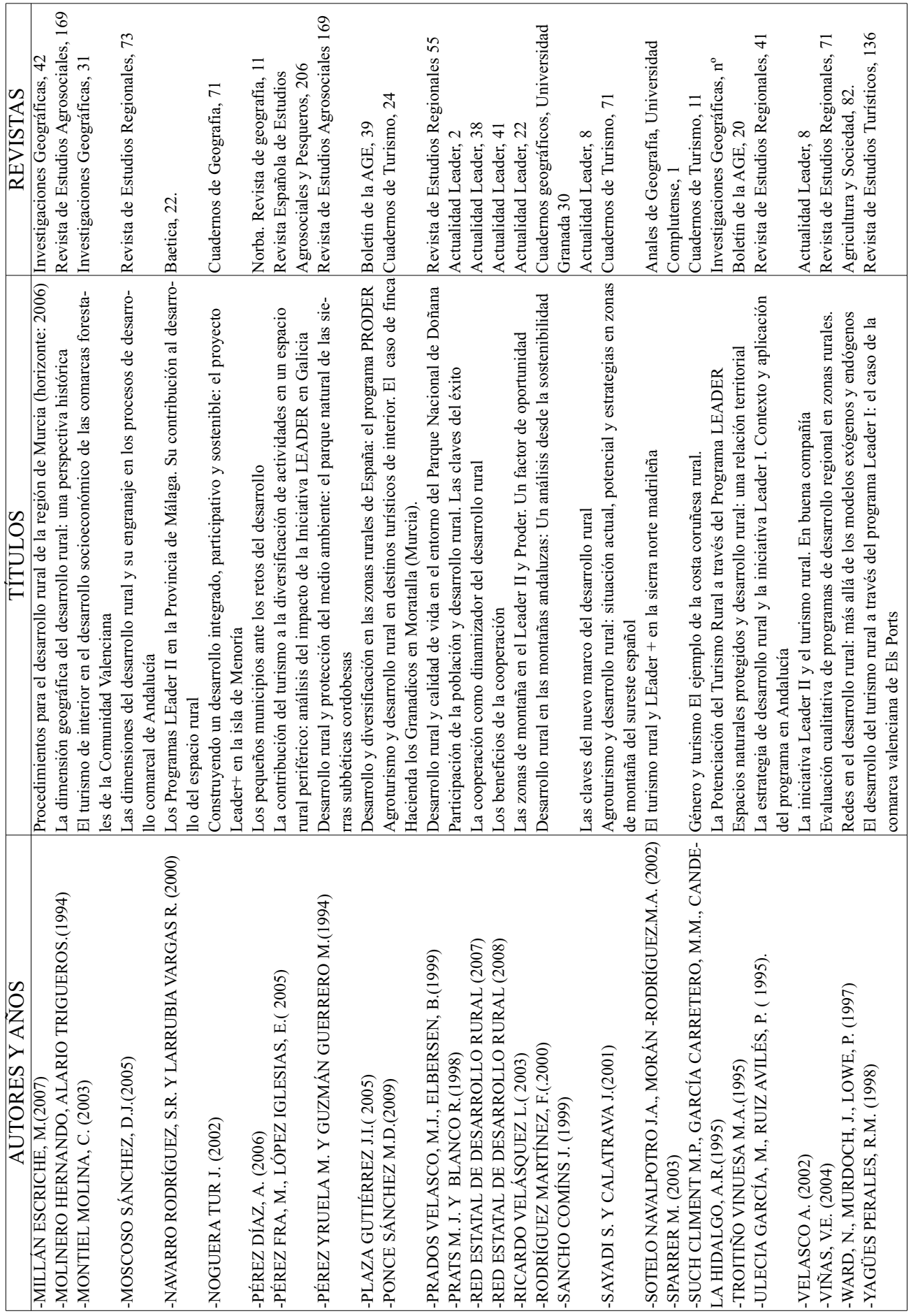

